\title{
Potentialité des zones humides infiltrant des eaux routières dans la nappe rhénane - étude de huit bassins de rétention et d'infiltration à Wolfisheim, Alsace
}

\author{
Stormwater basins as potential sources for road runoff \\ infiltration into the aquifer - case study of eight basins in \\ Wolfisheim, Alsace
}

\author{
Maximilien Nuel, Julien Laurent, Paul Bois, Pascale Chenon, Mohammed \\ Benbrahim, Georges Reeb, Fabien Toulet, Benoît Milliot, Emmanuel Belot et \\ Adrien Wanko
}

Volume 29, numéro 1, 2016

Reçu le 15 novembre 2014, accepté le 10 février 2015

URI : https://id.erudit.org/iderudit/1035712ar

DOI : https://doi.org/10.7202/1035712ar

Aller au sommaire du numéro

Éditeur(s)

Université du Québec - INRS-Eau, Terre et Environnement (INRS-ETE)

ISSN

1718-8598 (numérique)

Découvrir la revue

\section{Citer cet article}

Nuel, M., Laurent, J., Bois, P., Chenon, P., Benbrahim, M., Reeb, G., Toulet, F., Milliot, B., Belot, E. \& Wanko, A. (2016). Potentialité des zones humides infiltrant des eaux routières dans la nappe rhénane - étude de huit bassins de rétention et d'infiltration à Wolfisheim, Alsace. Revue des sciences de l'eau / Journal of Water Science, 29(1), 1-8. https://doi.org/10.7202/1035712ar

\section{Résumé de l'article}

Quatre filières de rétention et d'infiltration des eaux de ruissellement situées sur la route départementale 45 (RD 451) dans le Bas-Rhin constituent l'objet de l'étude. Chaque filière est composée : d'un bassin de rétention étanchéifié et équipé d'un " peigne végétal », d'un bassin d'infiltration végétalisé et équipé de plaques lysimétriques qui permettent de récupérer les eaux infiltrées à 25 et $50 \mathrm{~cm}$ de profondeur et d'un piézomètre/qualitomètre pour le suivi qualitatif et quantitatif de la nappe (mesure des concentrations en hydrocarbures aromatiques polycycliques (HAP) et en métaux). Enfin, une station météorologique in situ donne accès aux paramètres climatiques et permet ainsi de déterminer les flux de ruissellement, d'infiltration, d'évaporation, d'évapotranspiration et d'évapoconcentration. Suite aux différents épisodes pluvieux, la capacité d'infiltration des bassins perméables reste excellente, aucun colmatage n'ayant été observé. Les végétaux implantés se sont bien développés et leur rôle de filtre est constaté pour la fraction particulaire en surface. L'analyse de la qualité des eaux de surface et infiltrées, relevées mensuellement, met en évidence la présence de HAP et de métaux (aluminium, baryum et plomb). Les eaux de la nappe rhénane au droit du site étudié sont quant à elles dépourvues de HAP; cependant il est à noter la présence d'aluminium et de baryum, entre 30 et $130 \mu \mathrm{g} \cdot \mathrm{L}^{-1}$. Les prélèvements ont permis de mettre en évidence l'absence d'écotoxicité des eaux qui s'infiltrent en aval de la filière $n^{0} 3$ sur deux niveaux trophiques de l'écosystème souterrain, et sur des organismes d'eau de surface. Des préconisations méthodologiques et instrumentales sont faites afin d'accroître le champ des connaissances techniques et scientifiques concernant la structure, le rôle et le fonctionnement des bassins d'infiltration d'eaux routières ainsi que les interactions entre différentes matrices (sol, végétal et liquide) qui les composent. 


\section{POTENTIALITÉ DES ZONES HUMIDES INFILTRANT DES EAUX ROUTIÈRES DANS LA NAPPE RHÉNANE - ÉTUDE DE HUIT BASSINS DE RÉTENTION ET D’INFILTRATION À WOLFISHEIM, ALSACE}

Stormwater basins as potential sources for road runoff infiltration into the aquifer - case study of eight basins in Wolfisheim, Alsace

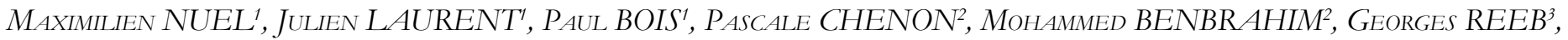
FABIEN TOULET', BENOÎT MILLIOT'5 ${ }^{5}$ EMMANUEL BELOT'5, ADRIEN WANKO ${ }^{* *}$

${ }^{1}$ ICube, UMR 7357, ENGEES, CNRS, Université de Strasbourg, 2, rue Boussingault, 67000 Strasbourg, France

${ }^{2}$ Rittmo Agroenvironnement, 37, rue de Herrlisheim, 68000 Colmar, France

${ }^{3}$ Atelier ReeB, 2, rue de Genève, 67000 Strasbourg, France

${ }^{4}$ Aprona, 140, rue du Logelbach, 68000 Colmar, France

${ }^{5}$ Conseil Général Bas Rhin, Place du Quartier Blanc, 67000 Strasbourg, France

Reçu le 15 novembre 2014, accepté le 10 février 2015

\section{RÉSUMÉ}

Quatre filières de rétention et d'infiltration des eaux de ruissellement situées sur la route départementale 45 (RD 451) dans le Bas-Rhin constituent l'objet de l'étude. Chaque filière est composée : d'un bassin de rétention étanchéifié et équipé d'un " peigne végétal ", d'un bassin d'infiltration végétalisé et équipé de plaques lysimétriques qui permettent de récupérer les eaux infiltrées à 25 et $50 \mathrm{~cm}$ de profondeur et d'un piézomètre/ qualitomètre pour le suivi qualitatif et quantitatif de la nappe (mesure des concentrations en hydrocarbures aromatiques polycycliques (HAP) et en métaux). Enfin, une station météorologique in situ donne accès aux paramètres climatiques et permet ainsi de déterminer les flux de ruissellement, d'infiltration, d'évaporation, d'évapotranspiration et d'évapoconcentration. Suite aux différents épisodes pluvieux, la capacité d'infiltration des bassins perméables reste excellente, aucun colmatage n'ayant été observé. Les végétaux implantés se sont bien développés et leur rôle de filtre est constaté pour la fraction particulaire en surface. L'analyse de la qualité des eaux de surface et infiltrées, relevées mensuellement, met en évidence la présence de HAP et de métaux (aluminium, baryum et plomb). Les eaux de la nappe rhénane au droit du site étudié sont quant à elles dépourvues de HAP; cependant il est à noter la présence d'aluminium et de baryum, entre 30 et $130 \mu \mathrm{g} \cdot \mathrm{L}^{-1}$. Les prélèvements ont permis de mettre en évidence l'absence d'écotoxicité des eaux qui s'infiltrent en aval de la filière $n^{\circ} 3$ sur deux niveaux trophiques de l'écosystème souterrain, et sur des organismes d'eau de surface. Des préconisations méthodologiques et instrumentales sont faites afin d'accroître le champ des connaissances techniques et scientifiques concernant la structure, le rôle et le fonctionnement des bassins d'infiltration d'eaux routières ainsi que les interactions entre différentes matrices (sol, végétal et liquide) qui les composent.

Mots-clés : Eaux routières, ruissellement, traitement, bassin d'infiltration, écotoxicité, HAP, métaux, plantes. 


\section{ABSTRACT}

Four stormwater basins located alongside a country road (Bas-Rhin, France) and designed to mitigate runoff road water pollution have been studied. Each system consists of a storage basin, designed to prevent infiltration and equipped with a "planted area", followed by a planted infiltration basin. Collection of infiltrating water $(25 \mathrm{~cm}$ and $50 \mathrm{~cm}$ depth) is achieved with draining lysimeters. Piezometers were set up to perform groundwater qualitative and quantitative analyses (Polycyclic aromatic hydrocarbons (PAH) and metal concentrations only). Climate parameters were measured in situ by a weather station, which allowed computation of runoff, infiltration, evaporation, evapotranspiration and evapoconcentration fluxes. No clogging could be observed after rain events, as shown by excellent infiltration capacity measurements. Plants developed well and the surface particulate phase was visually observed to be well sieved by this system. Monthly sampling revealed the presence of PAHs and metals (aluminium, barium and lead) in surface and infiltrated water, yet only aluminium and barium (30-130 $\left.\mu \mathrm{g} \cdot \mathrm{L}^{-1}\right)$ could be detected in groundwater right below the stormwater basins. Ecotoxicity tests performed on infiltrating water showed no adverse effects on two groundwater ecosystem trophic levels as well as surface water organisms. Methodological and technical guidelines are provided to enhance technical and scientific knowledge on road water infiltration systems. Structure, role, functioning of these systems as well as interactions among soil, plant and water within them could thus be better understood.

\section{Key Words: Road runoff, stormwater basin, treatment, infiltration, ecotoxicology, PAH, metals, plants.}

\section{INTRODUCTION}

Dans le contexte de l'amélioration de la qualité chimique et biologique des masses d'eau imposée par la Directive Cadre sur l'Eau (CE, 2000), le présent document réalise une étude diagnostique du fonctionnement des bassins de rétention et d'infiltration des eaux routières à travers un sous-sol perméable et sus-jacent à la nappe rhénane. Le site concerné par l'implantation des bassins est situé sur la Route Départementale 45 (RD 451) - Déviation de Wolfisheim et Oberschaeffolsheim au niveau de la bretelle de raccordement sur la Route Nationale 4 (N4) dans le Bas-Rhin.

Ce projet vise principalement à accroître le champ des connaissances techniques et scientifiques relatif à la problématique d'infiltration des eaux pluviales (PAGOTTO et LEGRETU, 1999; REVITT et al., 2004; MANGANI et al., 2005; CHOCAT et al., 2007; CITEAU, 2008;
BERNDTSSON, 2014) et particulièrement concernant la structure, le rôle et le fonctionnement des bassins d'infiltration d'eaux routières. Pour atteindre cet objectif, l'ENGEES, RITTMO Agroenvironnement, l'APRONA et l'ATELIER REEB ont décidé d'unir leurs compétences en matière de gestion hydraulique et environnementale des eaux urbaines et dans la mise en œuvre de projets collaboratifs à visée scientifique auprès de décideurs publics, en l'occurrence le Conseil Général du Bas-Rhin.

Cet article a pour but la présentation du site d'étude, la description des outils méthodologiques employés pour la quantification des flux, les suivis analytique, écotoxicologique et végétal suite à 12 mois d'études. C'est une approche transdisciplinaire qui a pour objectif à long terme : i) l'identification, la caractérisation, et la quantification des sources d'éléments traces et de métaux issus des eaux de ruissellement routières; ii) l'étude des mécanismes favorisant le transfert des polluants dans les compartiments eau, sol et plantes et leur dégradation physique, biologique et chimique; iii) la rédaction d'un guide technique de préconisation de conception et d'exploitation des systèmes de traitement des eaux de ruissellement routières.

\section{MATÉRIELS ET MÉTHODES}

\subsection{Le site d'étude}

Cette étude concerne quatre filières d'infiltration des eaux de ruissellement issues de la RD 45 (coordonnées GPS en Lambert 2 - filière $n^{\circ} 1: X=991023,65$ m et $Y=2411866,73$ m) qui récupèrent la totalité des eaux de voirie (Figure 1). L’hydrogéologie sous-jacente est constituée du Grand système aquifere Plio-Quaternaire de la plaine d'Alsace et du Grand domaine hydrogéologique Oligocène du Fossé Rhénan (SIGES Aquifère rhénan). Les profondeurs de la nappe au droit du site sont comprises entre 12 et $14 \mathrm{~m}$. Chaque filière est composée d'un bassin de rétention étanchéifié et équipé d'un "peigne végétal " puis d'un bassin d'infiltration végétalisé avec des espèces spécifiques (joncs des chaisiers, baldingères, iris des marais et roseaux). Les bassins de rétention sont dimensionnés pour une pluie biennale alors que les bassins d'infiltration le sont pour une pluie décennale, une voie de contournement permettant de protéger le premier bassin le cas échéant. Au fil de l'eau, les quatre filières suivies ont la même configuration hydraulique; l'agencement des différents ouvrages est mis en évidence dans la figure 2. L'analyse des coupes hydrogéologiques précise la couverture lœssique des alluvions sur le secteur qui varie de 14 $\mathrm{m}$ à l'ouest à $11,70 \mathrm{~m}$ à l'est. Le tableau 1 résume les principales caractéristiques des différents bassins. 


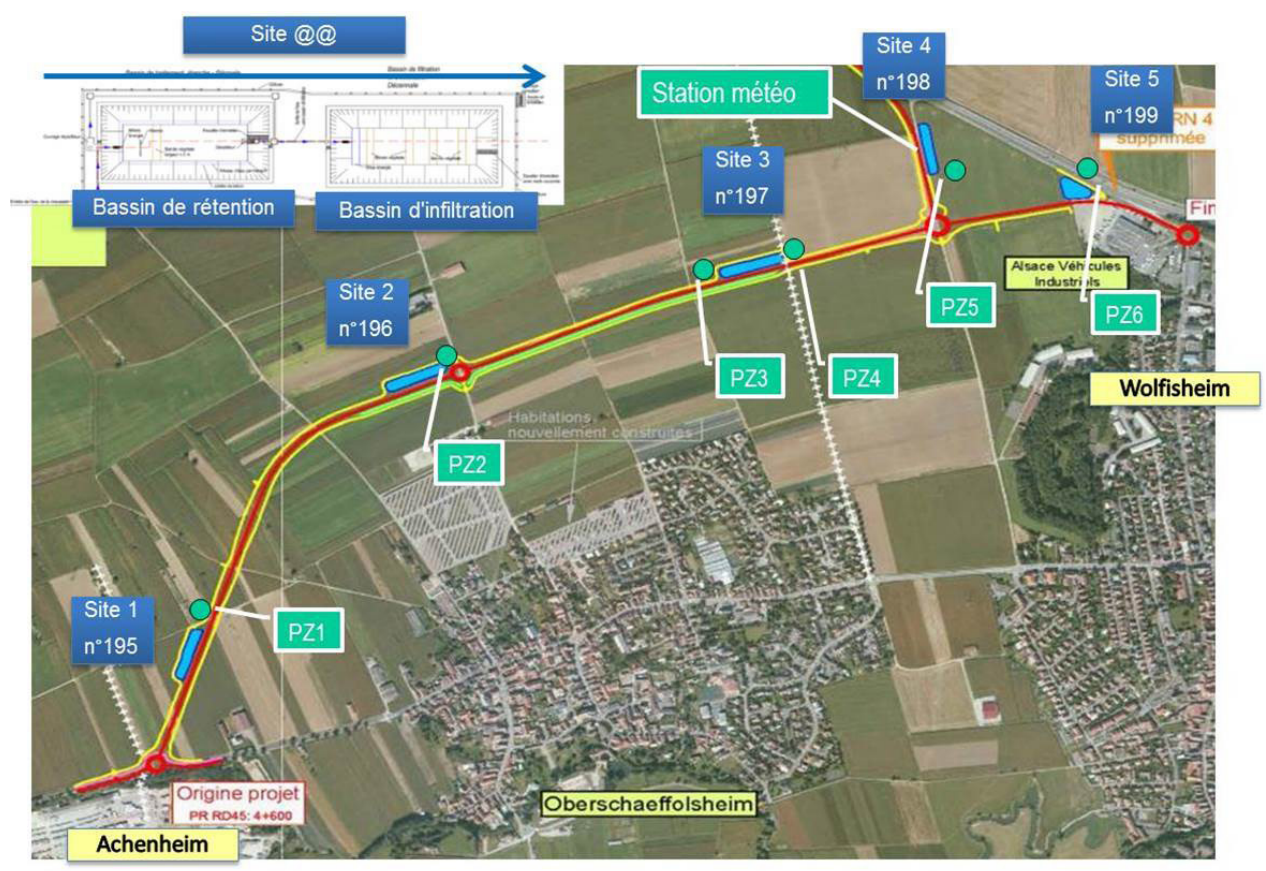

Figure 1. Bassins versants des sites de traitement des eaux de ruissellement. Corresponding catchment areas for each treatment site.

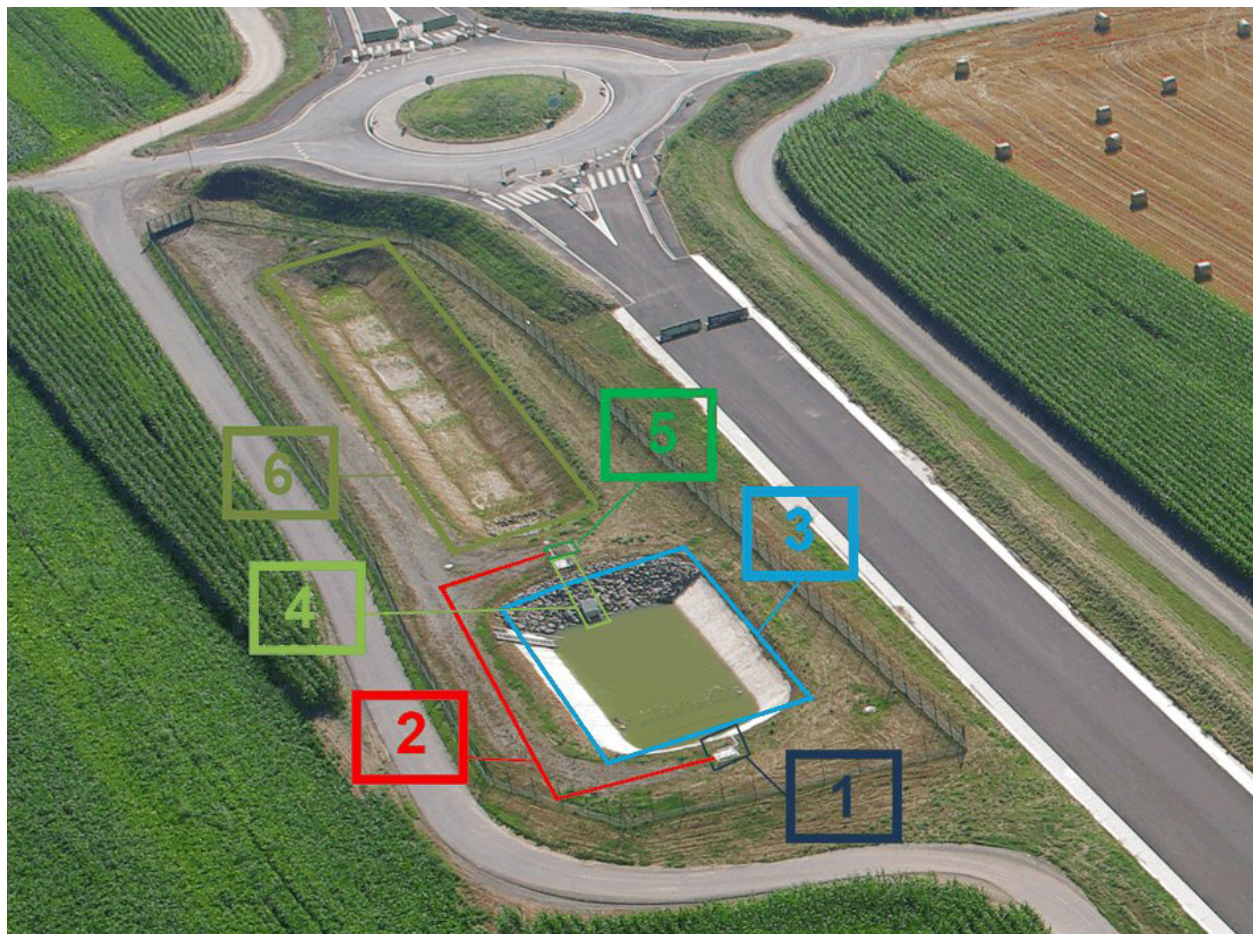

Figure 2. Disposition schématique des ouvrages suivis : 1) regard équipé d'un déversoir d'orage en entrée de station et recueillant les eaux polluées; 2 ) voie de contournement allant du déversoir d'orage au bassin imperméable; 3) premier bassin étanche recueillant les eaux de ruissellement routières; 4) regard équipé d'un dégrilleur en entrée et d'une vanne à clapet en sortie; 5) regard recueillant les eaux de l'ouvrage cité précédemment ainsi que les eaux issues de la voie de contournement; 6) bassin d'infiltration des eaux qui constitue l'exutoire de la zone humide.

Simplified description of studied systems: 1) Catch basin with a stormwater overflow at the inlet of the station that receives the polluted waters; 2) Bypass leading from the overflow to the impervious basin; 3) Initial impervious basin that receives road runoff; 4) Catch basin with a screen at its inlet and a backwater valve at its outlet; 5) Catch basin collecting the water from the preceding basin as well as the water coming from the bypass; 6) Infiltration basin that constitutes the outlet from the constructed wetland. 
Tableau 1. Dimension des bassins de rétention et d'infiltration étudiés.

Table 1. Dimensions of the stormwater basins.

\begin{tabular}{llccc}
\hline & Filière & Surface $\left(\mathbf{m}^{\mathbf{2}}\right)$ & Hauteur utile $(\mathbf{m})$ & Débit de fuite $\left(\mathbf{L} \cdot \mathbf{s}^{-1}\right)$ \\
\hline \multirow{2}{*}{1} & Bassin de rétention & 141 & 1,20 & 13 \\
& Bassin d'infiltration & 300 & 1,90 & Sans objet \\
2 & Bassin de rétention & 120 & 1,20 & 13 \\
& Bassin d'infiltration & 210 & 1,83 & Sans objet \\
3 & Bassin de rétention & 142 & 1,20 & 13 \\
& Bassin d'infiltration & 360 & 1,97 & Sans objet \\
\multirow{2}{*}{4} & Bassin de rétention & 102 & 1,20 & Sans objet \\
\hline
\end{tabular}

\subsection{Instrumentation du site}

Les bassins d'infiltration sont équipés de plaques lysimétriques qui permettent de récupérer les eaux infiltrées à 25 et $50 \mathrm{~cm}$ de profondeur. La collecte des eaux drainées par les plaques lysimétriques se fait via un bunker souterrain équipé de deux réceptacles plastiques d'une contenance de $30 \mathrm{~L}$ et situé à l'aval de chaque bassin d'infiltration. Un piézomètre/ qualitomètre $(\mathrm{PZ})$ installé sur chaque filière étudiée permet le suivi qualitatif et quantitatif des eaux souterraines à l'aval des filières. Une station météorologique in situ donne accès aux paramètres climatiques sur un pas de temps choisi et permet ainsi de déterminer les flux hydriques (ruissellement, infiltration, évaporation, évapotranspiration).

\subsection{Suivis quantitatif, qualitatif et écotoxicologique}

La figure 3 illustre les différents apports et pertes volumétriques. L'évaporation des bassins de décantation est approchée par la formule de Rohwer (1931) et l'évapotranspiration des bassins d'infiltration par la formule de Penman (1948). Par ailleurs, pour caractériser le comportement de la nappe dans le secteur d'étude, six piézomètres ont été installés dont trois sont équipés pour le suivi en continu des niveaux de la nappe; un à l'aval de chaque bassin et un à l'amont du bassin trois.

Concernant le suivi qualitatif, les campagnes de prélèvement sont réalisées mensuellement à différents points de chaque filière : en aval du bassin de décantation (numéro 4 sur la figure 2) et en aval du bassin d'infiltration (un échantillon composite des eaux drainées à 25 et $50 \mathrm{~cm}$ est réalisé). Le prélèvement des échantillons d'eaux souterraines donne l'état qualitatif initial de la nappe. Seules les concentrations en HAP et métaux sont mesurées sur l'ensemble de ces échantillons bruts non filtrés.

L'approche écotoxicologique proposée vise à vérifier que les eaux qui percolent n'auront pas d'impact écotoxique sur l'écosystème aquatique. L'évaluation a été menée à quatre moments de l'année sur les eaux d'infiltration en provenance des plaques lysimétriques à l'aide de tests d'écotoxicité aigus et chroniques sur différents bio-indicateurs : lentilles d'eau (effet sur la croissance), algues unicellulaires (effet sur la croissance) et deux microcrustacés (effet sur la survie et sur la reproduction).

\section{RÉSULTATS ET DISCUSSION}

\subsection{Evolution de la végétation}

Au stade actuel, les plantes se sont enracinées et ont bien passé le premier hiver (pas de fortes gelées, températures favorables à l'enracinement, apports d'eau largement suffisants). Un marnage d'environ $50 \mathrm{~cm}$ dans les différents bassins d'infiltration a pu être constaté sur les berges. Des rejets de saules se sont spontanément implantés sur le pourtour du bassin étanche $\mathrm{n}^{\circ} 1$ : ils présentent un risque de perforation $\mathrm{du}$ complexe d'étanchéité de ce bassin.

\subsection{Bilans hydriques}

L'étude des données météo a permis de mettre en évidence que les flux volumiques en entrée sont principalement dus aux ruissellements des chaussées (95\% du total) alors que les flux sortants sont quant à eux dus majoritairement à l'infiltration (l'évapotranspiration et l'évaporation ne représentent pas de volumes très importants). Le tableau 2 est une synthèse du suivi hydraulique dans le bassin d'infiltration de la filière $\mathrm{n}^{\circ} 1$. On observe une forte variabilité des volumes cumulés mensuels et des pertes mensuelles cumulées par évapotranspiration potentielle variant de 2 à $36,7 \mathrm{~m}^{3}$.

\subsection{Qualité des eaux de surfaces et des eaux souterraines}

Les résultats présentés au tableau 3 mettent en évidence des concentrations en aluminium relativement élevées pour 


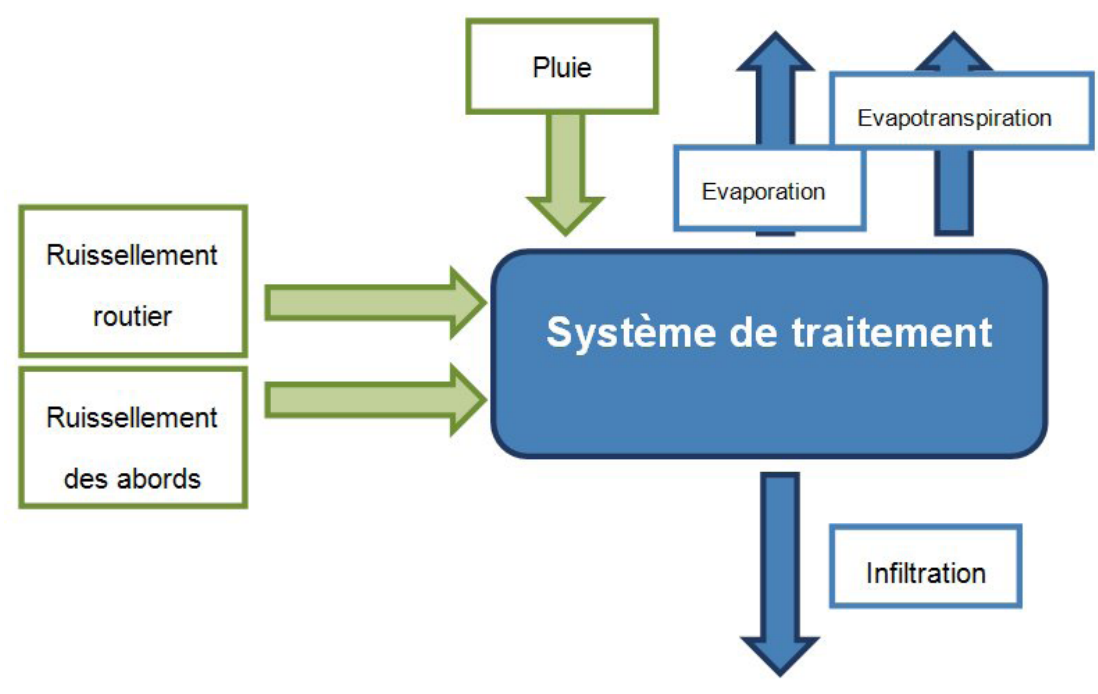

Figure 3. Bilan qualitatif des flux hydrauliques entrant et sortant des bassins. Theoretical mass balance for hydraulic fluxes.

Tableau 2. Synthèse des volumes ruisselés, précipités et évapotranspirés dans le bassin d'infiltration de la filière $\mathbf{n}^{\circ} 1$. Table 2. Synthesis of rain, runoff and evapotranspired flows in stormwater basin no. 1.

\begin{tabular}{|c|c|c|c|c|c|c|}
\hline \multirow[b]{2}{*}{ Mois } & \multicolumn{2}{|c|}{ Ruissellement } & \multicolumn{2}{|c|}{ Pluie directe } & \multicolumn{2}{|c|}{ Évapotranspiration } \\
\hline & $\begin{array}{c}\text { Cumul mensuel } \\
\left(\mathbf{m}^{3}\right)\end{array}$ & $\begin{array}{l}\text { Débit max } \\
\left(\mathbf{m}^{3} \cdot \mathbf{h}^{-1}\right)\end{array}$ & $\begin{array}{c}\text { Cumul mensuel } \\
\left(\mathbf{m}^{3}\right)\end{array}$ & $\begin{array}{l}\text { Débit max } \\
\left(\mathbf{m}^{3} \cdot \mathbf{h}^{-1}\right)\end{array}$ & $\begin{array}{c}\text { Cumul mensuel } \\
\left(\mathbf{m}^{3}\right)\end{array}$ & $\begin{array}{c}\text { Débit max } \\
\left(\mathbf{m}^{3} \cdot \mathbf{h}^{-1}\right)\end{array}$ \\
\hline Décembre 2013 & 333,88 & 33,29 & 9,93 & 0,99 & 2,08 & 0,06 \\
\hline Janvier 2014 & 334,89 & 25,22 & 9,96 & 0,75 & 6,24 & 0,08 \\
\hline Février 2014 & 356,07 & 38,33 & 10,59 & 1,14 & 10,66 & 0,13 \\
\hline Mars 2014 & 28,24 & 14,12 & 0,84 & 0,42 & 29,02 & 0,19 \\
\hline Avril 2014 & 211,83 & 68,59 & 6,30 & 2,04 & 36,69 & 0,25 \\
\hline $1-5$ mai 2014 & 230,99 & 20,17 & 6,72 & 0,60 & 14,31 & 0,25 \\
\hline
\end{tabular}

des eaux infiltrées. Une campagne de prélèvement a eu lieu sur deux jours afin de mesurer les polluants présents dans les eaux de la nappe phréatique. Aucune trace de HAP n'a été mesurée dans les eaux de la nappe. En revanche, d'après la figure 4, les eaux de nappe sont initialement chargées en aluminium et en baryum, et ce de manière systématique sur les quatre filières. La caractérisation des sols et sous-sols au droit du site ne suffit pas à renseigner sur l'origine de ces éléments. La connaissance précise des fonds géochimiques pourrait y participer. En effet, les concentrations en baryum des eaux issues de trois forages dans Wolfisheim sont de : $36 \mu \mathrm{g}(\mathrm{Ba}) \cdot \mathrm{L}^{-1}$ (en 2003) au lieu-dit
Rue de la Chapelle; $76 \mu \mathrm{g}(\mathrm{Ba}) \cdot \mathrm{L}^{1}$ (en 2009) au lieu-dit Rue de la Forêt; $82 \mu \mathrm{g}(\mathrm{Ba}) \cdot \mathrm{L}^{-1}$ (en 2003) au lieu-dit Woerth (portail ADES). S'agissant des ordres de grandeur de concentration en aluminium dans les eaux de surface et souterraines, elles sont élevées et d'autant plus surprenantes que la seule valeur disponible de concentration en aluminium des eaux souterraines de Wolfisheim est de l'ordre de $1 \mu \mathrm{g}(\mathrm{Al}) \cdot \mathrm{L}^{-1}$ au lieu-dit Rue de la Forêt (portail ADES). Aucune trace de HAP n'a été mesurée dans les eaux de la nappe, en effet la présence de HAP dans les bassins n'est pas systématique à toutes les campagnes; le tableau 4 présente les valeurs les plus élevées par bassin. 
Tableau 3. Relevé des concentrations extrêmes dans les eaux infiltrées.

Table 3. Extreme concentration values for infiltrated water.

\begin{tabular}{lcc}
\hline \multirow{2}{*}{ Paramètres } & \multicolumn{2}{c}{ Concentration $\left(\boldsymbol{\mu g} \cdot \mathbf{L}^{-\mathbf{1}}\right)$} \\
\cline { 2 - 3 } & Minimum & Maximum \\
\hline Aluminium & 1365 & 4023 \\
Baryum & 21,5 & 33,67 \\
Cadmium & $-{ }^{a}$ & $-{ }^{a}$ \\
Plomb & 1,40 & 1,90 \\
\hline
\end{tabular}

${ }^{a}$ Valeur inférieure à la limite de détection analytique.

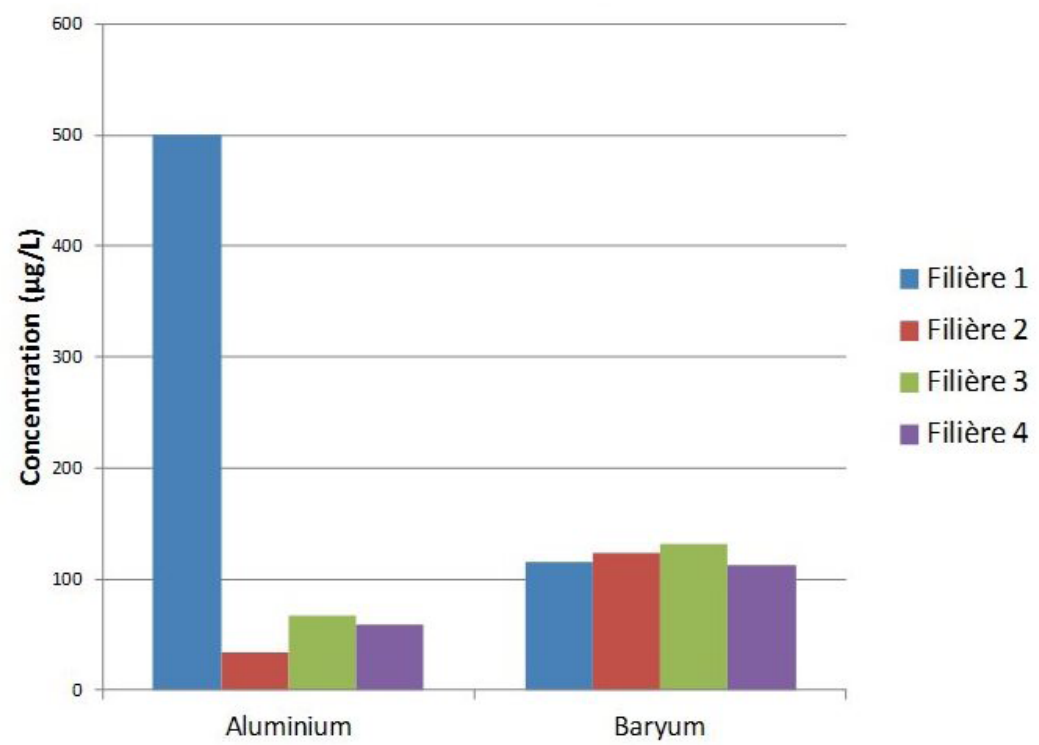

Figure 4. Concentration en métaux de la nappe rhénane au droit des filières étudiées.

Groundwater metal concentrations below the systems.

Tableau 4. Relevé des concentrations les plus élevées en HAP dans les eaux infiltrées.

Table 4. Highest PAH concentrations in infiltrated water.

\begin{tabular}{lccc}
\hline Bassin & $\begin{array}{c}\text { Nom polluant } \\
(\mathbf{H A P})\end{array}$ & $\begin{array}{c}\text { Concentration mesurée } \\
\left(\boldsymbol{\mu g} \cdot \mathbf{L}^{-1}\right)\end{array}$ & Date \\
\hline 1 & Benzo(ghi)pérylène & 0,283 & $13 / 03 / 2014$ \\
2 & 2-méthyl-naphtalène & 0,16 & $13 / 02 / 2014$ \\
3 & Benzo(b)fluoranthène & 0,112 & $14 / 04 / 2014$ \\
4 & Benzo(a)pyrène & 0,16 & $13 / 02 / 2014$ \\
\hline
\end{tabular}

\subsection{Impact sur le milieu récepteur}

Les trois premiers prélèvements ont permis de mettre en évidence l'absence d'écotoxicité des eaux qui s'infiltrent en aval de la filière $\mathrm{n}^{\circ} 3$ sur deux niveaux trophiques de l'écosystème aquatique souterrain, et sur des organismes d'eaux de surface (Figure 5). Aux plus fortes concentrations des eaux, les rotiferes (Brachionus calyciflorus) montrent une plus forte sensibilité que les autres bio-indicateurs. Les caractéristiques analytiques des eaux seront étudiées afin de mieux comprendre ce résultat.

\subsection{Suivi de la nappe}

Les premières mesures s'étalent sur sept mois pour le PZ1 (aval bassin $\mathrm{n}^{\circ} 1$ ) et le PZ3 (amont bassin $\mathrm{n}^{\circ} 3$ ) et quatre mois 
a
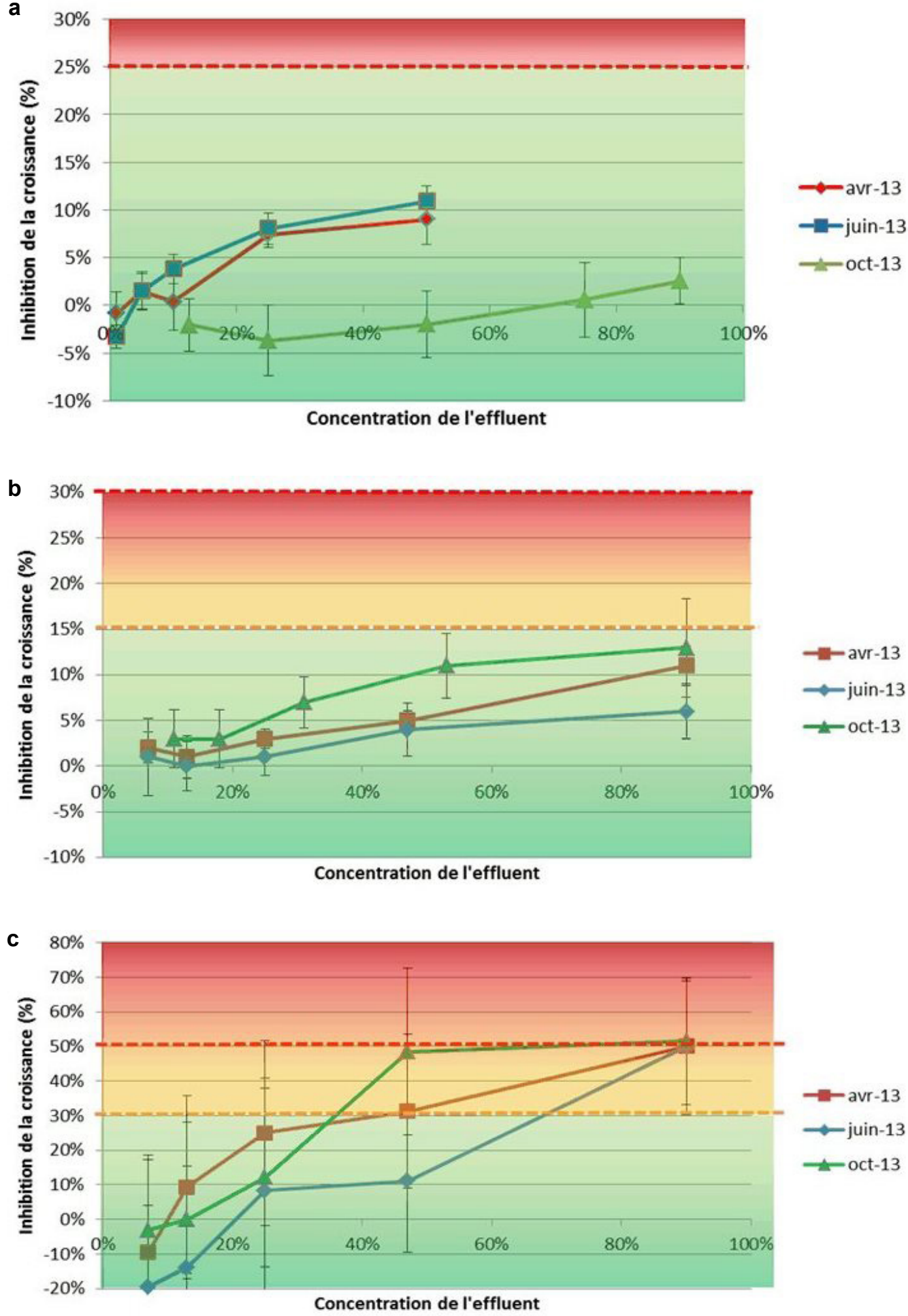

Figure 5. Inhibition de croissance des a) Lemna, b) algues et c) Brahionus des eaux d'infiltration en amont et en aval du bassin d'infiltration de la filière $n^{\circ} 3$.

Growth inbibition of a) Lemna, b) algae, and c) Brachionus by infiltration water up-and down-stream from system no. 3. 
pour le PZ6 (aval bassin $n^{\circ}$ 6). Les premiers résultats sont les suivants. i) Les profondeurs de la nappe sont comprises entre 12 et $14 \mathrm{~m}$ selon les sites. ii) les évolutions des niveaux de la nappe sur les trois sites équipés sont pour le moment très semblables. iii) Les variations du niveau piézométrique sont très faibles depuis le 31 juillet $2013(\approx 30 \mathrm{~cm})$. Ces battements sont parmi les plus faibles pour l'ensemble de la nappe d'Alsace. En comparaison, le battement moyen annuel de la nappe d'Alsace est proche de $2 \mathrm{~m}$. iv) Il n'y a pas de variation de température en lien avec le changement de saison (hiver/été).

\section{CONCLUSION}

Concernant la végétation implantée, les plantes se sont bien enracinées et commencent à assurer leur rôle de filtration. Par ailleurs, les trois premiers prélèvements ont permis de mettre en évidence l'absence d'écotoxicité des eaux qui s'infiltrent en aval du site $\mathrm{n}^{\circ} 3$ sur deux niveaux trophiques de l'écosystème, et sur des organismes d'eau de surface. Des effets écotoxiques ont été observés sur un rotifère (Brachionus calyciflorus). Enfin, la comparaison de la qualité des eaux avant et après infiltration semble montrer une efficacité épuratoire de l'infiltration tant sur les concentrations en métaux lourds que sur les HAP.

\section{REMERCIEMENTS}

La présence étude a été menée grâce au soutien du Conseil Départemental du Bas Rhin qui fait écho aux dispositions réglementaires en matière de protection de la qualité de l'eau. Le site expérimental étudié est un dispositif pilote à cette échelle en Alsace, cette démarche se veut être une première étape dans la perspective à moyen terme d'inscrire ces infrastructures dans un cadre pérenne.

\section{RÉFÉRENCES BIBLIOGRAPHIQUES}

Banque nationale d'Accès aux Données sur les Eaux Souterraines (ADES). www.ades.eaufrance.fr (consultation le 22 janvier 2015).

BERNDTSSON J.C. (2014). Storm water quality of first flush urban runoff in relation to different traffic characteristics. Urban Water J., 11, 284-296.

CHOCAT B., J.L. BERTRAND-KRAJEWSKI et S. BARRAUD (2007). Eaux pluviales urbaines et rejets urbains par temps de pluie. Techniques de l'ingénieur, W6800, 1-19.

CITEAU L. (2008). Transferteaux-sols-plantes de micropolluants: état des connaissances et application aux eaux de ruissellement urbaines. Rapport d'étude INRA, réalisé pour l'Agence de l'Eau Seine Normandie (AESN), France, 49 p.

MANGANI G., A. BERLONI, F. BELLUCCI, F. TATÀNO et M. MAIONE (2005). Evaluation of the pollutant content in road runoff first flush waters. Water Air Soil Pollut., 160, 213-228.

PAGOTTO C. et M. LEGRETU (1999). Evaluation of polluant loadings in the runoff water from a major rural highway. Sci. Total Environ., 235 (1-3), 143-50.

PENMAN H.L. (1948), Natural Evaporation from Open Water, Bare Soil and Grass, Proc. Royal Soc. London A, 193, 120-145.

REVITT D.M., R.B.E. SHUTES, R.H. JONES, M. FORSHAW et B. WINTER (2004). The performances of vegetative treatment systems for highway runoff during dry and wet conditions. Sci. Total Environ., 334, 261-270.

ROHWER C. (1931), Evaporation from Free Water Surface, USDA Tech. Bull., 217, 1-96. 